Mathematical Modelling and Analysis

Volume 21 Number 1, January 2016, 1-15

http://dx.doi.org/10.3846/13926292.2016.1127860

(C) Vilnius Gediminas Technical University, 2016
Publisher: Taylor\&Francis and VGTU

http://www.tandfonline.com/TMMA

ISSN: $1392-6292$

eISSN: 1648-3510

\title{
Study of Solutions to a Fourth Order Parabolic Equation*
}

\section{Bo Liang, Xiting Peng and Huiying Shen}

\author{
Dalian Jiaotong University, School of Science \\ 116028 Dalian, China \\ E-mail: cnliangbo@163.com
}

Received May 27, 2015; revised November 10, 2015; published online January 15, 2016

\begin{abstract}
This paper studies a fourth-order parabolic equation $u_{t}+\varepsilon\left(u^{n} u_{x x x}\right)_{x}-$ $\delta\left|u_{x x}\right|^{m} u_{x x}=0$ with the boundary conditions $u_{x x}=0, u=l$ and the initial condition $u(x, 0)=u_{0}(x)$. The existence of solutions is obtained from the semidiscretization method. When the initial function is close to a constant steady state solution, the uniqueness of the bounded solutions is obtained. Finally, by the iteration technique from its semi-discrete problem, the solution exponentially converges to a constant steady state solution as the time tends to infinity.
\end{abstract}

Keywords: fourth-order parabolic, existence, uniqueness, semidiscretization, large-time behavior.

AMS Subject Classification: 35G20; 35G30; 35G61; 35Q35.

\section{Introduction}

In recent years, the research of nonlinear fourth-order parabolic equations has become a hot topic. Specially, the typical models include the Cahn-Hilliard equation and the thin film equation, which can be used to describe the evolution of a conserved concentration field during phase separation and analyze the motion of a very thin layer of viscous incompressible fluids along an inclined plane, respectively.

The Cahn-Hilliard equation(see [9]) has the form $u_{t}+\nabla \cdot\left(k \nabla\left(\varepsilon^{2} \Delta u+\right.\right.$ $\left.\left.A^{\prime}(u)\right)\right)=0$ where the constants $k, A, \varepsilon^{2}$ denote the atomic mobility, the free energy, the parameter proportional to the interface energy respectively and $-\left(\varepsilon^{2} \Delta u+A^{\prime}(u)\right)$ is a kind of chemical potential. Elliott, Zheng and Garcke in the paper $[11,12]$ have studied this equation with a linear and a degenerate mobility respectively. Xu, Zhou in [18,19] and Liang, Zheng in [14] applied the semi-discrete method to get the existence and stability results to this kind of model with a gradient mobility.

\footnotetext{
* Supported by the NSF of China under grants (No.11201045).
} 
For the thin film equation, it can be described by a class of fourth order degenerate parabolic equations(see [15]):

$$
u_{t}+\left(m(u) u_{x x x}+f\left(u, u_{x}, u_{x x}\right)\right)_{x}=0
$$

with the function $m(u)=0$ when $u=0$. Appropriate forms have been applied in modeling fluid flows such as draining of foams and the movement of contact lenses. For example, the surface tension driven thin film flows can be modeled by using the following fourth order degenerate parabolic equation:

$$
\frac{\partial u}{\partial t}+\frac{\partial}{\partial x}\left(\frac{u^{3}}{3}\left(C h_{x x x}-\delta B h_{x} \cos \alpha+B \sin \alpha\right)+A \frac{u_{x}}{u}+\frac{M}{2} \sigma_{x} u^{2}\right)=0 .
$$

The first result in mathematics was given by Bernis and Friedman [3] to the equation $u_{t}+\left(u^{n} u_{x x x}\right)_{x}=0$ and they proved the existence and nonnegativity of weak solutions. The existence in the distributional sense and the long time decay were studied by Bertozzi and Pugh [5] for the thin film equation with a second-order diffusion term in the one-dimensional space. Boutat, Hilout, J. E. Rakotoson and J. M. Rakotoson [8] studied a generalized thin-film equation with period boundary in multidimensional space. Moreover, the paper [13] investigated the existence of the weak solutions and strong solutions with the initial function near a positive constant steady state solution. For other results, the readers may refer to the papers $[1,2,4,7,16]$.

For the semi-discretization method, the paper [10] has developed a unifying method to prove the existence and uniqueness of weak solutions for a nonuniformly parabolic equation. The reader may also see [13], [14] and [6] to know about the applications of semi-discretization method in the fourth order parabolic equations and the p-Laplacian equations. For this paper, we can obtain some accurate estimates by applying the semi-discretization method and we will show the effect of the second order nonlinear diffusion term for the fourth order parabolic equation.

In this paper, we mainly concern the following thin film model with a second-order diffusion term:

$$
\begin{aligned}
& u_{t}+\varepsilon\left(u^{n} u_{x x x}\right)_{x}-\delta\left|u_{x x}\right|^{m} u_{x x}=0 \text { in } Q_{T}, \\
& u_{x x}=0, u=l \text { on } \Gamma, \\
& u(x, 0)=u_{0}(x) \text { on } \Omega,
\end{aligned}
$$

where $\Omega=(0,1), Q_{T}=\Omega \times(0, T), \Gamma=\partial \Omega \times(0, T), n, \varepsilon, \delta, l$ are all positive constants and $m$ is a nonnegative constant. If letting $V=-u_{x x}$, the equation (1.1) can be considered as a second-order elliptic-parabolic system. The form is as the following

$$
\begin{aligned}
& u_{t}-\varepsilon\left(u^{n} V_{x}\right)_{x}+\delta|V|^{m} V=0 \text { in } Q_{T}, \\
& -u_{x x}=V \text { in } Q_{T} \\
& V=0, u=l \text { on } \Gamma \\
& u(x, 0)=u_{0}(x) \text { on } \Omega .
\end{aligned}
$$


Our elementary idea is to use the system (1.4)-(1.5) as a transition to get the existence and regularity for the equation (1.1). From the condition (1.6), we know that the solution $u$ is positive near the boundary $\partial \Omega$. Thus, we want to show that the positivity of the solution can be preserved if the initial function $u_{0}$ is close to the constant boundary $l$. In order to get the existence of the system (1.4)-(1.7), we will introduce a semi-discrete problem and construct a class of approximating solutions.

The weak solutions are shown as follow:

Theorem 1. (Existence) Let $\gamma$ be a positive constant such that $\left\|u_{0}-l\right\|_{H_{0}^{1}(\Omega)} \leq$ $\gamma<l$. Then there exists a positive weak solution of (1.4)-(1.7) satisfying

(1) $\frac{\partial u}{\partial t} \in L^{\frac{m+2}{m+1}}\left(0, T ; H^{-1}(\Omega)\right), u \in L^{\infty}\left(0, T ; H^{1}(\Omega)\right) \cap C\left([0, T] ; L^{2}(\Omega)\right), u \geq$ $l-\gamma>0, V \in L^{2}\left(0, T ; H_{0}^{1}(\Omega)\right), V \in L^{m+2}\left(Q_{T}\right)$;

(2) For each $\phi \in C_{0}^{\infty}\left(Q_{T}\right)$,

$$
\begin{aligned}
& \int_{0}^{T}\left\langle\frac{\partial u}{\partial t}, \phi\right\rangle d t+\varepsilon \iint_{Q_{T}} u^{n} V_{x} \phi_{x} d x d t+\delta \iint_{Q_{T}}|V|^{m} V \phi d x d t=0, \\
& -\iint_{Q_{T}} u_{x x} \phi d x d t=\iint_{Q_{T}} V \phi d x d t \\
& u(0)=u_{0}(x) \text { a.e. in } \Omega,
\end{aligned}
$$

where $<\cdot, \cdot>$ denotes the inner product between $H^{-1}$ and $H_{0}^{1}$.

Remark 1. By the condition $\left\|u_{0}-l\right\|_{H_{0}^{1}(\Omega)} \leq \gamma<l$ and the embedding $\| u_{0}-$ $l\left\|_{L^{\infty}(\Omega)} \leq\right\| u_{0}-l \|_{H_{0}^{1}(\Omega)}$, we have $u_{0} \geq l-\gamma>0$. It means that the method in Theorem 1 can not be applied in multidimensional space directly.

Substituting the relation $V=-u_{x x}$ into (1.4) in the sense of distribution, we can get the existence for the original equation (1.1).

Corollary 1. Suppose that the conditions of Theorem 1 hold and the estimate $\left\|u_{0}-l\right\|_{H_{0}^{1}(\Omega)} \leq \gamma<l$ is valid. Then there exists a weak solution of (1.1)-(1.3) satisfying

(1) $\frac{\partial u}{\partial t} \in L^{\frac{m+2}{m+1}}\left(0, T ; H^{-1}(\Omega)\right), u \in L^{\infty}\left(0, T ; H^{1}(\Omega)\right) \cap C\left([0, T] ; L^{2}(\Omega)\right)$, $u \geq l-\gamma>0, u_{x x} \in L^{2}\left(0, T ; H_{0}^{1}(\Omega)\right) \cap L^{m+2}\left(Q_{T}\right)$;

(2) For each $\phi \in C_{0}^{\infty}\left(Q_{T}\right)$,

$$
\begin{aligned}
& \int_{0}^{T}\left\langle\frac{\partial u}{\partial t}, \phi\right\rangle d t-\varepsilon \iint_{Q_{T}} u^{n} u_{x x x} \phi_{x} d x d t-\delta \iint_{Q_{T}}\left|u_{x x}\right|^{m} u_{x x} \phi d x d t=0, \\
& u(0)=u_{0}(x) \text { a.e. in } \Omega .
\end{aligned}
$$

If $\gamma$ (see Theorem 1) is proper small, we can get the uniqueness as following.

Theorem 2. (Uniqueness) The solution of (1.1)-(1.3) satisfying $\|u-l\|_{H_{0}^{1}(\Omega)} \leq$ $\gamma$ is unique for small $\gamma$.

In the following theorem, we will apply the entropy functional method to show that the solutions of (1.1)-(1.3) decay exponentially to the constant steady state $l$ in $H^{1}$-norm as $t \rightarrow \infty$. 
Theorem 3. (Exponential decay) Suppose that the conditions of Theorem 1 hold. Then $\|u-l\|_{H^{1}(\Omega)} \leq \rho_{0} e^{-C t}$, where $\rho_{0}=\int_{\Omega}\left(u_{0}-l\right)_{x}^{2} \mathrm{~d} x$.

The paper is arranged as follows. We will prove the existence of solutions in Section 2. The uniqueness will be established in Section 3. Finally, the asymptotic limit for $t \rightarrow \infty$ will be proved in Section 4 .

\section{Existence of solutions}

To prove the Theorem 1, it is necessary to solve the following semi-discrete problem

$$
\begin{aligned}
& \quad \frac{1}{h}\left(u_{k}-u_{k-1}\right)-\varepsilon\left(\left|u_{k-1}\right|^{n} V_{k x}\right)_{x}+\delta\left|V_{k}\right|^{m} V_{k}=0 \quad \text { in } \Omega, \\
& -u_{k x x}=V_{k} \quad \text { in } \Omega, \\
& u_{k}=l, \quad V_{k}=0 \quad \text { on } \partial \Omega,
\end{aligned}
$$

where $u_{k}=u(x, k h), V_{k}=V(x, k h), h=\frac{T}{N}$ and $k=1,2, \ldots, N$.

We prove the existence of $(2.1)-(2.3)$ at first.

Lemma 1. For given $u_{k-1} \in H^{2}(\Omega)$, there exists a unique weak solution $\left(u_{k}, V_{k}\right) \in H^{2}(\Omega) \times H^{2}(\Omega) \cap H_{0}^{1}(\Omega)$ of (2.1)-(2.3).

Proof. Firstly, we need to prove the existence of $u_{k}$ and $V_{k}$ for the problem (2.1)-(2.3) for given $u_{k-1}$. It is convenient to consider the following problem:

$$
\begin{aligned}
& \frac{1}{h}(u-w)-\varepsilon\left(|w|^{n} V_{x}\right)_{x}+\delta|V|^{m} V=0 \quad \text { in } \Omega, \\
& -u_{x x}=V \quad \text { in } \Omega, \\
& u=l, V=0 \quad \text { on } \partial \Omega,
\end{aligned}
$$

where $w$ is a known function and $\|w-l\|_{H_{0}^{1}(\Omega)} \leq \gamma$. The weak solution is defined as following. For any test function $\phi \in C_{0}^{\infty}(\Omega)$,

$$
\begin{aligned}
& \frac{1}{h} \int_{\Omega}(u-w) \phi d x+\varepsilon \int_{\Omega}|w|^{n} V_{x} \phi_{x} d x+\delta \int_{\Omega}|V|^{m} V \phi d x=0, \\
& -\int_{\Omega} u_{x x} \phi d x=\int_{\Omega} V \phi d x .
\end{aligned}
$$

In order to apply the fixed point theorem, we consider the following system:

$$
\begin{aligned}
& \sigma \frac{1}{h}(u-w)-\varepsilon\left(|w|^{n} V_{x}\right)_{x}+\delta|v|^{m} V=0 \quad \text { in } \Omega, \\
& -u_{x x}=\sigma v \quad \text { in } \Omega, \\
& u=l, \quad V=0 \quad \text { on } \partial \Omega,
\end{aligned}
$$

where $\sigma \in[0,1]$. For $v \in H_{0}^{1}(\Omega)$, there exists a unique strong solution $u \in$ $H^{2}(\Omega)$ by the equation (2.7). Next we will show that there exists a unique solution $V \in H^{2}(\Omega)$ for (2.6) and thus we introduce a bilinear functional

$$
a(V, \phi)=\varepsilon \int_{\Omega}|w|^{n} V_{x} \phi_{x} d x+\delta \int_{\Omega}|v|^{m} V \phi d x
$$


and a linear functional

$$
F(\phi)=-\sigma \frac{1}{h} \int_{\Omega}(u-w) \phi d x .
$$

By applying Hölder inequality and the boundedness of $w$, it is easy to show that

$$
\begin{aligned}
|a(V, \phi)| & \leq\left.\varepsilon\left|\int_{\Omega}\right| w\right|^{n} V_{x} \phi_{x} d x|+\delta| \int_{\Omega}|v|^{m} V \phi d x \mid \\
& \leq C\left\|V_{x}\right\|_{L^{2}(\Omega)}\left\|\phi_{x}\right\|_{L^{2}(\Omega)}+\delta\|V\|_{L^{2}(\Omega)}\|\phi\|_{L^{2}(\Omega)} \\
& \leq C\|V\|_{H_{0}^{1}(\Omega)}\|\phi\|_{H_{0}^{1}(\Omega)},
\end{aligned}
$$

which means $a(V, \phi)$ is bounded. Furthermore, it is coercive in that

$$
|a(V, V)|=\varepsilon \int_{\Omega}|w|^{n}\left|V_{x}\right|^{2} d x+\delta \int_{\Omega}|v|^{m}|V|^{2} d x \geq C\|V\|_{H_{0}^{1}(\Omega)}^{2} .
$$

On the basis of Lax-Milgram theorem, there exists a unique weak solution $V \in H_{0}^{1}(\Omega)$ for $(2.6)$ such that

$$
F(\phi)=a(V, \phi)
$$

Thus we can define the fixed point operator

$$
\begin{aligned}
T:[0,1] \times H_{0}^{1}(\Omega) & \longrightarrow H_{0}^{1}(\Omega), \\
(\sigma, v) & \longmapsto V .
\end{aligned}
$$

For all $v \in H_{0}^{1}(\Omega)$ satisfying $T(v, \sigma)=v$, we want to prove that $\|v\|_{H^{2}(\Omega)} \leq C$ here $C>0$ is independent of $v$ and $\sigma$. Using (2.7), we get $\|u\|_{H^{1}(\Omega)} \leq\|v\|_{L^{2}(\Omega)}$. Furthermore, by (2.6), we have the elliptic estimate

$$
\begin{aligned}
\|v\|_{H^{2}(\Omega)} & \leq C\left(1+\left\|\sigma \frac{1}{h}(u-w)+\delta|v|^{m} v\right\|_{L^{2}(\Omega)}\right) \\
& \leq C\left(1+\|v\|_{L^{2}(\Omega)}+\|v\|_{L^{2(m+1)}(\Omega)}^{m+1}\right) .
\end{aligned}
$$

On the other hand, by taking $-(u-l)_{x x}$ as a test function, we get

$$
\frac{1}{2 h} \int_{\Omega}(u-l)_{x}^{2} d x+\varepsilon \int_{\Omega}|w|^{n}\left|v_{x}\right|^{2} d x+\delta \int_{\Omega}|v|^{m+2} d x \leq \frac{1}{2 h} \int_{\Omega}(w-l)_{x}^{2} d x .
$$

Thus, one has $\|v\|_{L^{2}(\Omega)}+\|v\|_{L^{2(m+1)}(\Omega)}^{m+1} \leq C$ by the embedding $H^{1}(\Omega) \hookrightarrow$ $L^{\infty}(\Omega)$ and then we have $\|v\|_{H^{2}(\Omega)} \leq C$. With the help of the compact embed$\operatorname{ding} H^{2}(\Omega) \hookrightarrow H_{0}^{1}(\Omega), T$ is continuous and compact. Leray-Schauder's fixed point theorem ensures the existence of (2.6)-(2.8).

Furthermore, according to (2.9), we have

$$
\|u-l\|_{H^{1}(\Omega)} \leq \gamma,
$$

and so by the Sobolev imbedding theorem $H^{1}(\Omega) \hookrightarrow C^{\alpha}(\bar{\Omega})$ for some $\alpha \in(0,1)$, it follows that

$$
u \geq l-\gamma>0
$$


Now for given $u_{k-1} \in H^{2}(\Omega)$ and $\left\|u_{k-1}-l\right\|_{H^{1}(\Omega)} \leq \gamma$, the semi-discrete problem (2.1)-(2.3) admits a solution

$$
\left(u_{k}, V_{k}\right) \in H^{2}(\Omega) \times\left(H^{2}(\Omega) \cap H_{0}^{1}(\Omega)\right) .
$$

Besides, we can conclude that $u_{k-1}, u_{k}$ and $V_{k}$ also satisfy the weak form (2.4)$(2.5)$, i. e.

$$
\begin{aligned}
& \quad \frac{1}{h} \int_{\Omega}\left(u_{k}-u_{k-1}\right) \phi d x+\varepsilon \int_{\Omega}\left|u_{k-1}\right|^{n} V_{k x} \phi_{x} d x+\delta \int_{\Omega}\left|V_{k}\right|^{m} V_{k} \phi d x=0, \\
& -\int_{\Omega} u_{k x x} \phi d x=\int_{\Omega} V_{k} \phi d x
\end{aligned}
$$

for any test function $\phi \in C_{0}^{\infty}(\Omega)$.

Finally, we prove the uniqueness. Let $\left(u_{k}, V_{k}\right)$ and $\left(u_{k}^{\prime}, V_{k}^{\prime}\right)$ be two different solutions of (2.1)-(2.3). Taking $\left(u_{k}-u_{k}^{\prime}\right)_{x x}=-\left(V_{k}-V_{k}^{\prime}\right)$ as a test function in the difference of the corresponding equations, we get

$$
\begin{gathered}
\frac{1}{h} \int_{\Omega}\left|\left(u_{k}-u_{k}^{\prime}\right)_{x}\right|^{2} d x+\varepsilon \int_{\Omega}\left|u_{k-1}\right|^{n}\left|\left(V_{k}-V_{k}^{\prime}\right)_{x}\right|^{2} d x \\
+\delta \int_{\Omega}\left(\left|V_{k}\right|^{m} V_{k}-\left|V_{k}^{\prime}\right|^{m} V_{k}^{\prime}\right)\left(V_{k}-V_{k}^{\prime}\right) d x=0 .
\end{gathered}
$$

By the inequality $\left(|x|^{m} x-|y|^{m} y\right)(x-y) \geq 0$ with any real numbers $x, y$, we have the uniqueness.

In order to obtain the existence of solutions, we need to define some approximate solutions:

$$
\begin{aligned}
& w^{(N)}(x, t)=\sum_{k=1}^{N} \chi_{k}(t) u_{k}(x), \quad \widetilde{w}^{(N)}(x, t)=\sum_{k=1}^{N} \chi_{k}(t) u_{k-1}(x), \\
& u^{(N)}(x, t)=\sum_{k=1}^{N} \chi_{k}(t)\left[\lambda_{k}(t) u_{k}(x)+\left(1-\lambda_{k}(t)\right) u_{k-1}(x)\right], \\
& V^{(N)}(x, t)=\sum_{k=1}^{N} \chi_{k}(t) V_{k}(x),
\end{aligned}
$$

where $\chi_{k}(t)$ is a characteristic function on the time interval $((k-1) h, k h]$ $(k=1, \ldots, n)$ and $\lambda_{k}(t)= \begin{cases}t / h-(k-1), & \text { if } t \in((k-1) h, k h], \\ 0, & \text { otherwise. }\end{cases}$

In the following, we establish some uniform estimates for those approximation solutions.

Lemma 2. The following uniform estimates hold for $w^{(N)}, V^{(N)}$ and $u^{(N)}$ :

$$
\begin{aligned}
& \left\|w^{(N)}\right\|_{L^{\infty}\left(0, T ; H^{1}(\Omega)\right)}+\left\|V^{(N)}\right\|_{L^{2}\left(0, T ; H_{0}^{1}(\Omega)\right)}+\left\|\frac{\partial u^{(N)}}{\partial t}\right\|_{L^{\frac{m+2}{m+1}}\left(0, T ; H^{-1}(\Omega)\right)} \\
& +\left\|u^{(N)}\right\|_{L^{\infty}\left(0, T ; H^{1}(\Omega)\right)}+\left\|V^{(N)}\right\|_{L^{m+2}\left(Q_{T}\right)} \leq C .
\end{aligned}
$$


Furthermore, there exists a subsequence(not relabeled) and two functions $u$ and $\xi$ such that the following limits can be performed:

$$
\begin{aligned}
& w^{(N)} \stackrel{*}{\rightarrow} u \quad \text { weakly* in } L^{\infty}\left(0, T ; H^{1}(\Omega)\right), \\
& V^{(N)} \rightarrow V \quad \text { in } L^{2}\left(0, T ; H_{0}^{1}(\Omega)\right) \cap L^{m+2}\left(Q_{T}\right), \\
& \left|V^{(N)}\right|^{m} V^{(N)} \rightarrow \xi \quad \text { in } L^{\frac{m+2}{m+1}}\left(Q_{T}\right), \\
& \frac{\partial u^{(N)}}{\partial t} \rightarrow \frac{\partial u}{\partial t} \quad \text { weakly in } L^{\frac{m+2}{m+1}}\left(0, T ; H^{-1}(\Omega)\right), \\
& u^{(N)} \stackrel{*}{\rightarrow} u \quad \text { weakly* in } L^{\infty}\left(0, T ; H^{1}(\Omega)\right), \\
& u^{(N)} \rightarrow u \quad \text { strongly in } C\left([0, T] ; L^{2}(\Omega)\right), \quad u^{(N)} \rightarrow u \quad \text { a.e. in } Q_{T} .
\end{aligned}
$$

Proof. By (2.10)-(2.11), we get

$$
\begin{aligned}
& \frac{1}{h} \sum_{k=1}^{N} \int_{(k-1) h}^{k h} \int_{\Omega} \chi_{k}(t)\left(u_{k}-u_{k-1}\right) \phi \mathrm{d} x d t+\varepsilon \sum_{k=1}^{N} \int_{(k-1) h}^{k h} \\
& \quad \times \int_{\Omega} \chi_{k}(t)\left|u_{k-1}\right|^{n} V_{k x} \phi_{x} d x d t+\delta \sum_{k=1}^{N} \int_{(k-1) h}^{k h} \int_{\Omega} \chi_{k}(t)\left|V_{k}\right|^{m} V_{k} \phi d x d t=0 .
\end{aligned}
$$

Taking $\phi=V_{k}=-u_{k x x}$ yields

$$
\begin{aligned}
& -\sum_{k=1}^{N} \int_{\Omega}\left(u_{k}-u_{k-1}\right) u_{k x x} d x+\varepsilon \iint_{Q_{T}}\left|\widetilde{w}^{(N)}\right|^{n}\left|V_{x}^{(N)}\right|^{2} d x d t \\
& \quad+\delta \iint_{Q_{T}}\left|V^{(N)}\right|^{m+2} d x d t=0
\end{aligned}
$$

and then

$$
\begin{aligned}
& \int_{\Omega}\left|\left(u_{N}-l\right)_{x}\right|^{2} d x+\varepsilon \iint_{Q_{T}}\left|\widetilde{w}^{(N)}\right|^{n}\left|V_{x}^{(N)}\right|^{2} d x d t \\
& \quad+\delta \iint_{Q_{T}}\left|V^{(N)}\right|^{m+2} d x d t \leq \int_{\Omega}\left|\left(u_{0}-l\right)_{x}\right|^{2} m d x .
\end{aligned}
$$

On the other hand, for any $(x, t)$, we get

$$
\widetilde{w}^{(N)}(x, t)=\sum_{k=1}^{N} \chi_{k}(t) u_{k-1}(x) \geq l-\gamma
$$

and then

$$
\left\|V^{(N)}\right\|_{L^{2}\left(0, T ; H_{0}^{1}(\Omega)\right)} \leq C,\left\|V^{(N)}\right\|_{L^{m+2}\left(Q_{T}\right)} \leq C .
$$

For any $t \in(0, T)$, there exists some $k \in\{1, \ldots, n\}$ such that, for $t \in((k-$ 1) $h, k h]$, one has

$$
\left\|w_{x}^{(N)}(x, t)\right\|_{L^{2}(\Omega)}^{2}=\left\|u_{k x}(x)\right\|_{L^{2}(\Omega)}^{2} \leq C .
$$


It implies

$$
\left\|w^{(N)}\right\|_{L^{\infty}\left(0, T ; H^{1}(\Omega)\right)} \leq C .
$$

By the definition of $u^{(N)}(x, t)$, it is easy to get

$$
\frac{\partial u^{(N)}}{\partial t}=\frac{1}{h} \sum_{k=1}^{N} \chi_{k}\left(u_{k}-u_{k-1}\right)
$$

and then

$$
\begin{aligned}
& \int_{0}^{T}\left|\left\langle\frac{\partial u^{(N)}}{\partial t}, \phi\right\rangle\right| d t=\int_{0}^{T}\left|\left\langle\frac{1}{h} \sum_{k=1}^{N} \chi_{k}\left(u_{k}-u_{k-1}\right), \phi\right\rangle\right| d t \\
& \leq\left. C \int_{0}^{T}\left|\sum_{k=1}^{N} \chi_{k}(t) \int_{\Omega}\right| u_{k-1}\right|^{n} V_{k x} \phi_{x} d x\left|d t+C \int_{0}^{T}\right| \sum_{k=1}^{N} \chi_{k}(t) \int_{\Omega}\left|V_{k}\right|^{m} V_{k} \phi d x \mid d t \\
& \leq C \int_{0}^{T}\left(\int_{\Omega}\left|V_{x}^{(N)} \phi_{x}\right| d x\right) d t+\left.C \int_{0}^{T}\left|\int_{\Omega}\right| V^{(N)}\right|^{m} V^{(N)} \phi d x \mid d t \leq C,
\end{aligned}
$$

where $\phi$ is any test function in the space $L^{m+2}\left(0, T ; H_{0}^{1}(\Omega)\right)$. It follows that

$$
\left\|\frac{\partial u^{(N)}}{\partial t}\right\|_{L^{\frac{m+2}{m+1}}\left(0, T ; H^{-1}(\Omega)\right)} \leq C .
$$

On the basis of the definition of $u^{(N)}$, we have

$$
\begin{aligned}
& \left\|u^{(N)}\right\|_{L^{q}\left(0, T ; H_{0}^{1}(\Omega)\right)}^{q} \leq C^{q} \int_{0}^{T}\left(\int_{\Omega}\left|u_{x}^{(N)}\right|^{2} d x\right)^{\frac{q}{2}} d t \\
& =C^{q} \int_{0}^{T}\left(\int_{\Omega}\left|\sum_{k=1}^{n} \chi_{k}(t)\left[\lambda_{k}(t) u_{k x}(x)+\left(1-\lambda_{k}(t)\right) u_{(k-1) x}(x)\right]\right|^{2} d x\right)^{\frac{q}{2}} d t \\
& =C^{q} \sum_{k=1}^{N} \int_{(k-1) h}^{(k h)}\left(\int_{\Omega}\left|\left[\lambda_{k}(t) u_{k x}(x)+\left(1-\lambda_{k}(t)\right) u_{(k-1) x}(x)\right]\right|^{2} d x\right)^{\frac{q}{2}} d t \\
& \leq 2^{\frac{q}{2}} C^{q} \sum_{k=1}^{N} h\left(\int_{\Omega}\left(\left|u_{k x}(x)\right|^{2}+\left|u_{k-1 x}(x)\right|^{2}\right) d x\right)^{\frac{q}{2}} \leq 2^{\frac{q}{2}} C^{q} C_{1}^{\frac{q}{2}} T
\end{aligned}
$$

with $C>0$ independent of $q>1$, where we have applied the fact $\lambda_{k}(t) \in[0,1]$ and the estimate (2.9). Thus we have

$$
\left\|u^{(N)}\right\|_{L^{\infty}\left(0, T ; H_{0}^{1}(\Omega)\right)}=\lim _{q \rightarrow \infty}\left\|u^{(N)}\right\|_{L^{q}\left(0, T ; H_{0}^{1}(\Omega)\right)} \leq C .
$$

By the preceding energy estimates, there exists a subsequence of $w^{(N)}$ such that

$$
\begin{aligned}
& w^{(N) \stackrel{*}{\rightarrow}} u \quad \text { weakly* in } L^{\infty}\left(0, T ; H^{1}(\Omega)\right), \\
& V^{(N)} \rightarrow V \quad \text { in } L^{2}\left(0, T ; H_{0}^{1}(\Omega)\right)
\end{aligned}
$$


as $N \rightarrow \infty$. By Aubin's lemma [17] and the embedding $H^{1}(\Omega) \stackrel{\text { compact }}{\hookrightarrow} L^{\frac{m+2}{m+1}}(\Omega) \hookrightarrow H^{-1}(\Omega)$, we can infer the existence of a subsequence of $u^{(N)}$ such that, as $N \rightarrow \infty$,

$$
\begin{aligned}
& \frac{\partial u^{(N)}}{\partial t} \rightarrow \frac{\partial \rho}{\partial t} \quad \text { weakly in } L^{\frac{m+2}{m+1}}\left(0, T ; H^{-1}(\Omega)\right), \\
& u^{(N)} \stackrel{*}{\rightarrow} \rho \quad \text { weakly* in } L^{\infty}\left(0, T ; H^{1}(\Omega)\right), \\
& u^{(N)} \rightarrow \rho \quad \text { strongly in } C\left([0, T] ; L^{2}(\Omega)\right), \\
& u^{(N)} \rightarrow \rho \quad \text { a.e. in } Q_{T}, \quad\left|V^{(N)}\right|^{m} V^{(N)} \rightarrow \xi \quad \text { in } L^{\frac{m+2}{m+1}}\left(Q_{T}\right) .
\end{aligned}
$$

Finally, we need to show $\rho=u$. For any $\phi \in C_{0}^{\infty}\left(Q_{T}\right)$, we have

$$
\begin{aligned}
& \left|\int_{0}^{T} \int_{\Omega}\left(w^{(N)}-u^{(N)}\right) \varphi d x d t\right| \\
& =\left|\int_{0}^{T} \int_{\Omega} \sum_{k=1}^{N} \chi_{k}(t)\left(1-\lambda_{k}(t)\right)\left(u_{k}-u_{k-1}\right) \varphi d x d t\right| \\
& \leq \sum_{k=1}^{N} \int_{0}^{T} \chi_{k}(t) h\left(\left.\left|\int_{\Omega}\right| u_{k-1}\right|^{n} V_{k x} \phi_{x} d x|+| \int_{\Omega}\left|V_{k}\right|^{m} V_{k} \phi d x \mid\right) d t \\
& \leq C h\left(\iint_{Q_{T}}\left|V_{x}^{(N)} \phi_{x}\right| d x+\int_{\Omega}\left|V^{(N)}\right|^{m}\left|V^{(N)} \phi\right| d x\right) d t \leq C h \rightarrow 0
\end{aligned}
$$

as $N \rightarrow \infty$. It has $\rho=u$ a.e. in $Q_{T}$.

Lemma 3. It follows that

$$
\iint_{Q_{T}}\left(\widetilde{w}^{(N)}\right)^{n} V_{x}^{(N)} \phi_{x} d x d t \rightarrow \iint_{Q_{T}} u^{n} V_{x} \phi_{x} d x d t
$$

and

$$
\xi=|V|^{m} V \text { a.e. in } Q_{T}
$$

for any test function $\phi$.

Proof. Taking $V_{k}-V_{k-1}=-\left(u_{k}-u_{k-1}\right)_{x x}$ as a test function, we get

$$
\begin{gathered}
\frac{1}{h} \int_{\Omega}\left|\left(u_{k}-u_{k-1}\right)_{x}\right|^{2} d x+\varepsilon \int_{\Omega}\left|u_{k-1}\right|^{n} V_{k x}\left(V_{k}-V_{k-1}\right)_{x} d x \\
+\delta \int_{\Omega}\left|V_{k}\right|^{m} V_{k}\left(V_{k}-V_{k-1}\right) d x=0,
\end{gathered}
$$

which implies

$$
\begin{aligned}
& \sum_{k=1}^{N} \int_{\Omega}\left|\left(u_{k}-u_{k-1}\right)_{x}\right|^{2} d x \leq\left. C h \sum_{k=1}^{N}\left|\int_{\Omega}\right| u_{k-1}\right|^{n} V_{k x}\left(V_{k}-V_{k-1}\right)_{x} d x \mid \\
& \quad+\left.C h \sum_{k=1}^{N}\left|\int_{\Omega}\right| V_{k}\right|^{m} V_{k}\left(V_{k}-V_{k-1}\right) d x \mid \leq C T .
\end{aligned}
$$


Therefore, we get

$$
\begin{gathered}
\iint_{Q_{T}}\left|w^{(N)}-u^{(N)}\right|^{2} d x d t \leq h \sum_{k=1}^{N} \int_{\Omega}\left|u_{k}-u_{k-1}\right|^{2} d x \\
\leq C h \sum_{k=1}^{N} \int_{\Omega}\left|\left(u_{k}-u_{k-1}\right)_{x}\right|^{2} d x \leq C h .
\end{gathered}
$$

Letting $N \rightarrow \infty$, we have

$$
w^{(N)} \rightarrow u \text { strongly in } L^{2}\left(Q_{T}\right) .
$$

On the other hand, we will prove that $\widetilde{w}^{(N)} \rightarrow u$ strongly in the sense of $L^{2}\left(Q_{T}\right)$. A direct calculation shows that

$$
\begin{gathered}
\iint_{Q_{T}}\left|\widetilde{w}^{(N)}-w^{(N)}\right|^{2} d x d t=\iint_{Q_{T}}\left|\sum_{k=1}^{N} \chi_{k}(t)\left(u_{k}-u_{k-1}\right)\right|^{2} d x d t \\
\leq h \sum_{k=1}^{N} \int_{\Omega}\left|u_{k}-u_{k-1}\right|^{2} d x \leq C T h .
\end{gathered}
$$

Hence, by the mean value theorem, we obtain

$$
\begin{aligned}
\left|\iint_{Q_{T}}\left(\left(\widetilde{w}^{(N)}\right)^{n}-u^{n}\right) V_{x}^{(N)} \phi_{x} d x d t\right| & =\left|\iint_{Q_{T}} n \vartheta^{n-1}\left(\widetilde{w}^{(N)}-u\right) V_{x}^{(N)} \phi_{x} d x d t\right| \\
& \leq C\left\|\widetilde{w}^{(N)}-u\right\|_{L^{2}\left(Q_{T}\right)}\left\|V_{x}^{(N)} \phi_{x}\right\|_{L^{2}\left(Q_{T}\right)},
\end{aligned}
$$

where $\vartheta$ is between $u$ and $\widetilde{w}^{(N)}$ and it is bounded from the boundedness of $\widetilde{w}^{(N)}$ and $u$. Here $\widetilde{w}^{(N)}$ is bounded since, for any $q>1$,

$$
\begin{aligned}
& \left(\iint_{Q_{T}}\left|\widetilde{w}^{(N)}\right|^{q} d x d t\right)^{\frac{1}{q}}=\left(\iint_{Q_{T}}\left|\sum_{k=1}^{N} \chi_{k}(t) u_{k-1}(x)\right|^{q} d x d t\right)^{\frac{1}{q}} \\
& \leq\left(\sum_{k=1}^{N} \int_{(k-1) h}^{k h} \int_{\Omega}\left|u_{k-1}\right|^{q} d x d t\right)^{\frac{1}{q}} \leq T^{\frac{1}{q}}\left(\int_{\Omega}\left|u_{k-1}\right|^{q} d x\right)^{\frac{1}{q}} \leq C T^{\frac{1}{q}},
\end{aligned}
$$

and then by letting $q \rightarrow \infty$, we have $\widetilde{w}^{(N)} \in L^{\infty}\left(Q_{T}\right)$.

Now we can perform the limit $N \rightarrow \infty$ to get

$$
\left|\iint_{Q_{T}}\left(\left(\widetilde{w}^{(N)}\right)^{n}-u^{n}\right) V_{x}^{(N)} \phi_{x} \mathrm{~d} x \mathrm{~d} t\right| \rightarrow 0 .
$$

Thus we obtain

$$
\begin{aligned}
& \left|\iint_{Q_{T}}\left(\left(\widetilde{w}^{(N)}\right)^{n} V_{x}^{(N)}-u^{n} V_{x}\right) \phi_{x} d x d t\right| \\
& =\left|\iint_{Q_{T}}\left(\left(\widetilde{w}^{(N)}\right)^{n} V_{x}^{(N)}-u^{n} V_{x}^{(N)}+u^{n} V_{x}^{(N)}-u^{n} V_{x}\right) \phi_{x} d x d t\right| \\
& \leq\left|\iint_{Q_{T}}\left(\left(\widetilde{w}^{(N)}\right)^{n} V_{x}^{(N)}-u^{n} V_{x}^{(N)}\right) \phi_{x} d x d t\right|+\left|\iint_{Q_{T}}\left(u^{n} V_{x}^{(N)}-u^{n} V_{x}\right) \phi_{x} d x d t\right| \\
& \quad \rightarrow 0 .
\end{aligned}
$$


Now for each $\phi \in C_{0}^{\infty}\left(Q_{T}\right)$, we can perform the limit $N \rightarrow \infty$ in the form

$$
\begin{gathered}
\int_{0}^{T}\left\langle\frac{\partial u^{(N)}}{\partial t}, \phi\right\rangle d t+\varepsilon \iint_{Q_{T}} \widetilde{w}^{n} V_{x}^{(N)} \phi_{x} d x d t \\
+\delta \iint_{Q_{T}}\left|V^{(N)}\right|^{m} V^{(N)} \phi d x d t=0, \\
-\iint_{Q_{T}} u_{x x}^{(N)} \phi d x d t=\iint_{Q_{T}} V^{(N)} \phi d x d t
\end{gathered}
$$

to get

$$
\begin{aligned}
& \int_{0}^{T}\left\langle\frac{\partial u}{\partial t}, \phi\right\rangle \mathrm{d} t+\varepsilon \iint_{Q_{T}} u^{n} V_{x} \phi_{x} \mathrm{~d} x \mathrm{~d} t+\delta \iint_{Q_{T}} \xi \phi \mathrm{d} x \mathrm{~d} t=0, \\
& -u_{x x}=V \text { a.e. in } Q_{T} .
\end{aligned}
$$

By taking $\phi=V^{(N)}=-u_{x x}^{(N)}$ in $(2.12)$, we have

$$
\begin{aligned}
& \frac{1}{2} \int_{\Omega}\left|w_{x}^{(N)}(x, T)\right|^{2} d x-\frac{1}{2} \int_{\Omega}\left|u_{0 x}\right|^{2} d x+\varepsilon \iint_{Q_{T}} \widetilde{w}^{n}\left|V_{x}^{(N)}\right|^{2} d x d t \\
& +\delta \iint_{Q_{T}}\left|V^{(N)}\right|^{m+2} d x d t=0 .
\end{aligned}
$$

Besides, from the fact

$$
\iint_{Q_{T}}\left(\left|V^{(N)}\right|^{m} V^{(N)}-|(V-\varepsilon \phi)|^{m}(V-\varepsilon \phi)\right)\left(V^{(N)}-(V-\varepsilon \phi)\right) d x d t \geq 0,
$$

we have

$$
\begin{aligned}
& \delta \iint_{Q_{T}}\left|V^{(N)}\right|^{m+2} d x d t-\delta \iint_{Q_{T}}\left|V^{(N)}\right|^{m} V^{(N)}(V-\varepsilon \phi) d x d t \\
& \quad-\delta \iint_{Q_{T}}|V-\varepsilon \phi|^{m}(V-\varepsilon \phi) V^{(N)} d x d t+\delta \iint_{Q_{T}}|V-\varepsilon \phi|^{m+2} d x d t \geq 0 .
\end{aligned}
$$

Substituting (2.16) into (2.17) and taking $N \rightarrow \infty$, we get

$$
\begin{aligned}
& \frac{1}{2} \int_{\Omega}\left|u_{x}(x, T)\right|^{2} d x-\frac{1}{2} \int_{\Omega}\left|u_{0 x}\right|^{2} d x+\varepsilon \iint_{Q_{T}} u^{n}\left|V_{x}\right|^{2} d x d t \\
& \quad+\delta \iint_{Q_{T}} \xi(V-\varepsilon \phi) d x d t+\delta \iint_{Q_{T}}|V-\varepsilon \phi|^{m}(V-\varepsilon \phi) V d x d t \\
& \quad-\delta \iint_{Q_{T}}|V-\varepsilon \phi|^{m+2} d x d t \leq 0 .
\end{aligned}
$$

Taking $\phi=V=-u_{x x}$ in (2.14) and applying (2.18), we have

$$
\iint_{Q_{T}}\left(|V-\varepsilon \phi|^{m}(V-\varepsilon)-\xi\right) \phi d x d t \leq 0 .
$$

By letting $\varepsilon \rightarrow 0$ and the arbitrariness of $\phi$, one has $\xi=|V|^{m} V$ a.e. in $Q_{T}$.

From Lemma 1-Lemma 3, we can prove the results of Theorem 1 and Corollary 1. 


\section{Uniqueness}

In this section, we prove the uniqueness and then gives the proof of Theorem 3 .

It follows from the conditions of Theorem 2, any solutions $u$ of (1.1) have the lower bound $(l-\gamma)>0$. Using $u_{x x}$ as a test function in (1.1), we get

$$
\frac{1}{2} \int_{\Omega} u_{x}^{2}(x, T) d x+\varepsilon \iint_{Q_{T}} u^{n}\left|u_{x x x}\right|^{2} d x d t+\delta \iint_{Q_{T}}\left|u_{x x}\right|^{m+2} d x d t=\frac{1}{2} \int_{\Omega} u_{0 x}^{2} d x,
$$

and then

$$
\iint_{Q_{T}}\left|u_{x x x}\right|^{2} d x d t \leq \frac{C \gamma^{2}}{\varepsilon(l-\gamma)^{n}}
$$

where the constant $C$ is independent of $\gamma$.

Assume that $v$ is another solution which also satisfying (3.1) and let $w=$ $u-v$. Taking $w_{x x}$ as a test function in the differential equations for $u, v$, we obtain, for any $0<t<T$,

$$
\begin{aligned}
& \frac{1}{2} \int_{\Omega} w_{x}^{2}(x, t) d x+\varepsilon(l-\gamma)^{n} \iint_{Q_{T}} w_{x x x}^{2} d x d t+\varepsilon \iint_{Q_{T}}\left(u^{n}-v^{n}\right) v_{x x x} w_{x x x} d x d t \\
& \quad+\delta \iint_{Q_{T}}\left(\left|u_{x x}\right|^{m} u_{x x}-\left|v_{x x}\right|^{m} v_{x x}\right)\left(u_{x x}-v_{x x}\right) d x d t=0 .
\end{aligned}
$$

In view of the mean value theorem, we have for $n \geq 1$

$$
\begin{aligned}
& \left|\varepsilon \iint_{Q_{T}}\left(u^{n}-v^{n}\right) v_{x x x} w_{x x x} d x d t\right| \leq C \varepsilon \iint_{Q_{T}}\left|u-v \| v_{x x x}\right|\left|w_{x x x}\right| d x d t \\
& \quad \leq \frac{\varepsilon(l-\gamma)^{n}}{2} \iint_{Q_{T}} w_{x x x}^{2} d x d t+\frac{C \varepsilon}{(l-\gamma)^{n}} \iint_{Q_{T}}|u-v|^{2}\left|v_{x x x}\right|^{2} d x d t
\end{aligned}
$$

and for $0<n<1$

$$
\begin{gathered}
\left|\varepsilon \iint_{Q_{T}}\left(u^{n}-v^{n}\right) v_{x x x} w_{x x x} d x d t\right| \leq C \varepsilon(l-\gamma)^{n-1} \iint_{Q_{T}}\left|u-v \| v_{x x x}\right|\left|w_{x x x}\right| d x d t \\
\leq \frac{\varepsilon(l-\gamma)^{n}}{2} \iint_{Q_{T}} w_{x x x}^{2} d x d t+\frac{C \varepsilon}{l-\gamma} \iint_{Q_{T}}|u-v|^{2}\left|v_{x x x}\right|^{2} d x d t .
\end{gathered}
$$

Hence, for all $n>0$ and $0<t<T$, we have

$$
\begin{aligned}
& \frac{1}{2} \int_{\Omega} w_{x}^{2}(x, t) d x+\frac{\varepsilon(l-\gamma)^{n}}{2} \iint_{Q_{T}} w_{x x x}^{2} d x d t \\
& \leq \frac{C \varepsilon}{\Theta(l-\gamma)} \iint_{Q_{T}}|u-v|^{2}\left|v_{x x x}\right|^{2} d x d t \\
& \quad \leq \frac{C \varepsilon}{\Theta(l-\gamma)} \sup _{0<t<T} \int_{\Omega} w_{x}^{2} d x \iint_{Q_{T}}\left|v_{x x x}\right|^{2} d x d t
\end{aligned}
$$


where we have used the embedding $H^{1}(\Omega) \hookrightarrow C^{0}(\bar{\Omega})$ and the function $\Theta(s)=\left\{\begin{array}{ll}s^{n}, & n \geq 1 ; \\ s, & 0<n<1 .\end{array}\right.$ Since the solution $v$ satisfies $(3.1)$, we have

$$
\begin{aligned}
& \sup _{0<t<T} \int_{\Omega} w_{x}^{2}(x, t) d x \leq \frac{C \varepsilon}{\Theta(l-\gamma)} \sup _{0<t<T} \int_{\Omega} w_{x}^{2} d x \iint_{Q_{T}}\left|v_{x x x}\right|^{2} \mathrm{~d} x d t \\
& \leq \frac{C \gamma^{2}}{(l-\gamma)^{n} \Theta(l-\gamma)} \sup _{0<t<T} \int_{\Omega} w_{x}^{2}(x, t) d x
\end{aligned}
$$

We can conclude that $w_{x} \equiv 0$ for small $\gamma$. This yields the uniqueness.

\section{Exponential decay}

In this section, we prove the long time behavior under the conditions of Theorem 2. Taking $V_{k}=-\left(u_{k}-l\right)_{x x}$ as a test function in $(2.10)-(2.11)$, we get

$$
\begin{aligned}
& \frac{1}{2 h} \int_{\Omega}\left(u_{k}-l\right)_{x}^{2} d x+\varepsilon \int_{\Omega}\left|u_{k-1}\right|^{n}\left|u_{k x x x}\right|^{2} d x+\delta \int_{\Omega}\left|u_{k x x}\right|^{m+2} d x \\
& \quad \leq \frac{1}{2 h} \int_{\Omega}\left(u_{k-1}-l\right)_{x}^{2} d x .
\end{aligned}
$$

Apply $u_{k-1}>l-\gamma>0$ to have

$$
\frac{1}{2 h} \int_{\Omega}\left(u_{k}-l\right)_{x}^{2} d x \leq-C \int_{\Omega}\left|u_{k x x x}\right|^{2} d x+\frac{1}{2 h} \int_{\Omega}\left(u_{k-1}-l\right)_{x}^{2} d x,
$$

where $C$ is independent of $k$. Poincaré inequality yields

$$
\frac{1}{2 h} \int_{\Omega}\left(u_{k}-l\right)_{x}^{2} d x \leq-C \int_{\Omega}\left(u_{k}-l\right)_{x}^{2} d x+\frac{1}{2 h} \int_{\Omega}\left(u_{k-1}-l\right)_{x}^{2} d x .
$$

Letting

$$
\rho_{k}=\int_{\Omega}\left(u_{k}-l\right)_{x}^{2} d x, \quad k=0, \cdots, N
$$

we can establish an iteration

$$
\rho_{k} \leq \frac{1}{1+C h} \rho_{k-1}
$$

and then for any $t \in((k-1) h, k h]$,

$$
\rho_{k} \leq \rho_{0}(1+C h)^{-k} \leq \rho_{0}(1+C h)^{-t / h}, \quad k=0, \cdots, N .
$$

The definition of $w^{(N)}$ implies

$$
\begin{aligned}
\int_{\Omega}\left(w^{(N)}-l\right)_{x}^{2} d x & =\int_{\Omega}\left(\sum_{k=1}^{N} \chi_{k}(t) u_{k}-l\right)_{x}^{2} d x \\
& =\int_{\Omega}\left(u_{k}-l\right)_{x}^{2} \mathrm{~d} x=\rho_{k} \leq \rho_{0}(1+C h)^{-t / h}
\end{aligned}
$$


Applying the results of Section 3 and the weak lower semi-continuity for the space $H^{1}$, we obtain the exponential decay

$$
\int_{\Omega}(u-l)_{x}^{2} \mathrm{~d} x \leq \rho_{0} e^{-C t}
$$

as $h \rightarrow 0$ (i.e. $N \rightarrow \infty$ ).

\section{References}

[1] L. Ansini and L. Giacomelli. Doubly nonlinear thin-film equations in one space dimension. Archive for Rational Mechanics Analysis, 173(1):89-131, 2004. http://dx.doi.org/10.1007/s00205-004-0313-x.

[2] E. Beretta, M. Bertsch and R. Dal Passo. Nonnegative solutions of a fourthorder nonlinear degenerate parabolic equation. Archive for Rational Mechanics Analysis, 129(2):175-200, 1995. http://dx.doi.org/10.1007/BF00379920.

[3] F. Bernis and A. Friedman. Higher order nonlinear degenerate parabolic equations. J. Differential Equations, 83(1):179-206, 1990.

[4] A.L. Bertozzi and M.Pugh. The lubrication approximation for thin viscous films: regularity and long time behavior of weak solutions. Comm. Pure Appl. Math, 49(2):85-123, 1996. http://dx.doi.org/10.1002/(SICI)1097-0312(199602)49:2.

[5] A.L. Bertozzi and M. Pugh. The lubrication approximation for thin viscous films: the moving contact line with a 'porous media' cut-off of van der Waals interactions. Nonlinearity, 7(6):1535-1564, 1994. http://dx.doi.org/10.1088/0951$7715 / 7 / 6 / 002$.

[6] V. Bhuvaneswari, L. Shangerganesh and K. Balachandran. Weak solutions for $p$-Laplacian equation. Adv. Nonlinear Anal., 1(4):319-334, 2012. http://dx.doi.org/10.1515/anona-2012-0009.

[7] P.M. Bleher, J.L. Lebowitz and E.R. Speer. Existence and positivity of solutions of a fourth-order nonlinear PDE describing interface fluctuations. Comm. Pure. Appl. Math., 47:923-942, 1994.

[8] M. Boutat, S. Hilout, J.E. Rakotoson and J.M. Rakotoson. A generalized thin-film equation in multidimensional space. Nonlinear Analysis: Theory, Methods \& Applications, 69(4):1268-1286, 2008. http://dx.doi.org/10.1016/j.na.2007.06.028.

[9] J. M. Cahn and J. E. Hilliard. Free energy of a non-uniform system i. interfacial free energy. J. Chem. Phys., 28:258-267, 1958.

[10] Y. Cai and S. Zhou. Existence and uniqueness of weak solutions for a nonuniformly parabolic equation. Journal of Functional Analysis, 257(10):30213042, 2009. http://dx.doi.org/10.1016/j.jfa.2009.08.007.

[11] C. M. Elliott and H. Garcke. On the Cahn-Hilliard equation with degenerate mobility. SIAM Journal on Mathematical Analysis, 27(2):404-423, 1996.

[12] C. M. Elliott and S. Zheng. On the Cahn-Hilliard equation. Arch. Rational Mech. Anal., 96:339-357, 1986. http://dx.doi.org/10.1007/BF00251803.

[13] B. Liang. Existence and asymptotic behavior of solutions to a thin film equation with Dirichlet boundary. Nonlinear Analysis: Real World Applications, 12(3):1828-1840, 2011. http://dx.doi.org/10.1016/j.nonrwa.2010.11.014. 
[14] B. Liang and S. Zheng. Existence and asymptotic behavior of solutions to a nonlinear parabolic equation of fourth order. Journal of Mathematical Analysis and Applications, 348(1):234-243, 2008. http://dx.doi.org/10.1016/j.jmaa.2008.07.022.

[15] T.G. Myers. Thin films with high surface tension. SIAM Review, 40(3):441-462, 1998. http://dx.doi.org/10.1137/S003614459529284X.

[16] R. Dal Passo, H. Garcke and G. Grün. On a fourth-order degenerate parabolic equation: global entropy estimates, existence, and qualitative behavior of solutions. SIAM journal on mathematical analysis, 29(2):321-342, 1998. http://dx.doi.org/10.1137/S0036141096306170.

[17] J. Simon. Compact sets in the space $l^{p}(0, t ; b)$. Annali di Mathematica pura ed applicata, 146(4):65-96, 1987.

[18] M. Xu and S. Zhou. Existence and uniqueness of weak solutions for a generalized thin film equation. Nonlinear Analysis: Theory, Methods \& Applications, 60(4):755-774, 2005. http://dx.doi.org/10.1016/j.na.2004.01.013.

[19] M. Xu and S. Zhou. Stability and regularity of weak solutions for a generalized thin film equation. Journal of Mathematical Analysis and Applications, 337(1):49-60, 2008. http://dx.doi.org/10.1016/j.jmaa.2007.03.075. 